Supporting Information

\title{
Nanostructured and selective filter to improve detection of arsenic on surface plasmon nanosensors
}

\author{
Yulieth C. Reyes ${ }^{1}$, Luis Emerson $\mathrm{Coy}^{2}$, Luis Yate $^{3}$, Stefan Jurga ${ }^{2,4}$ and Edgar E. González ${ }^{1 *}$ \\ ${ }^{1}$ Pontificia Universidad Javeriana, Faculty of Engineering, Instituto Geofisico, Bogotá, 110231, Colombia \\ ${ }^{2}$ NanoBioMedical Centre, Adam Mickiewicz University, 61614 Poznan, Poland \\ ${ }^{3}$ CIC biomaGUNE, Paseo Miramón 182, 20009, San Sebastián, Spain \\ ${ }^{4}$ Department of Macromolecular Physics, Adam Mickiewicz University, 85 Umultowska str., 61-614 Poznan, \\ Poland \\ Corresponding Author: egonzale@javeriana.edu.co
}

\section{Table of Contents:}

- Transmittance Spectra

- Concentration of suspended solids and Arsenide pre/post cotton filter

- Absorbance Spectra

$\mathrm{p}(\mathrm{S}-4)$ 
S1

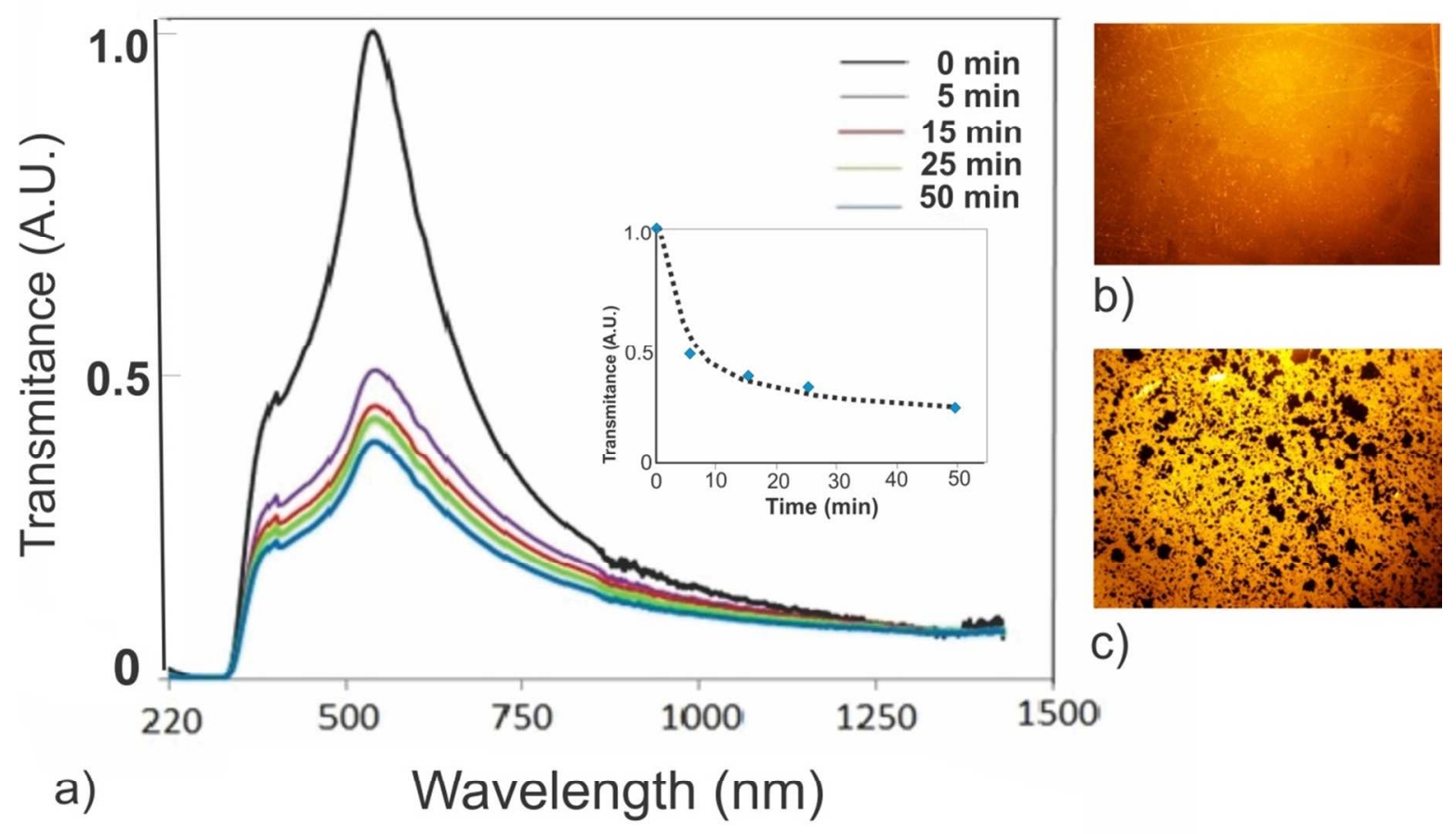

Figure S1. a) Transmittance spectra to the sensor surface exposed to water flow taken from station pt. 2 to times $0,5,15,25$ and 50 minutes respectively. In the inner box the temporal evolution of the transmittance is indicated. b) Optical photograph (x40) of gold surface without interaction with the water sample. c) Optical photograph (x40) of gold surface exposed for $20 \mathrm{~min}$ to a water sample station pto 2 . 


\section{Supporting Information}

S2

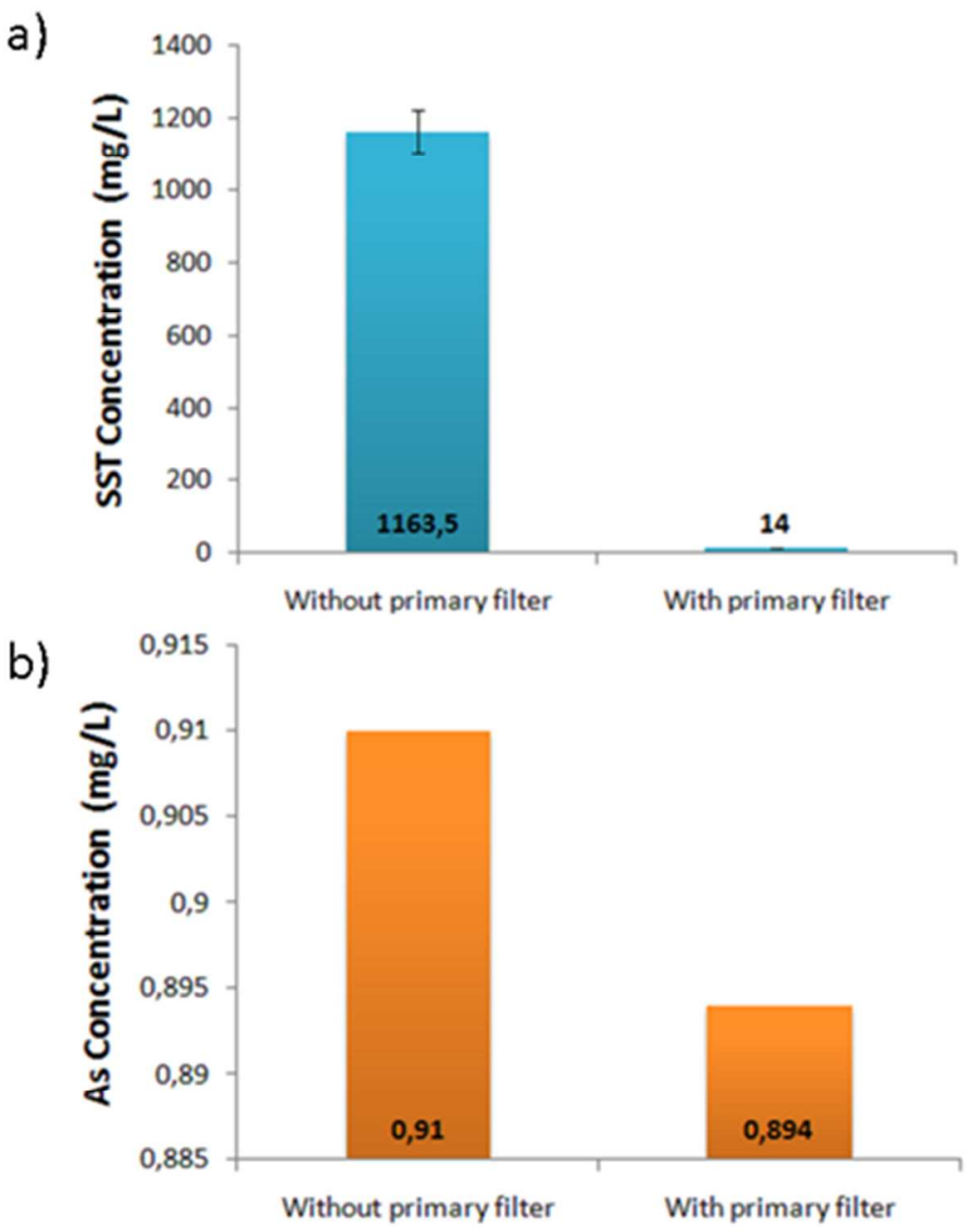

Figure S2. a) Bar chart for the concentration of suspended solids, before and after using the filter cotton. b) Bar chart for the concentration of As, before and after using the filter cotton. 


\section{Supporting Information}

\section{S3}

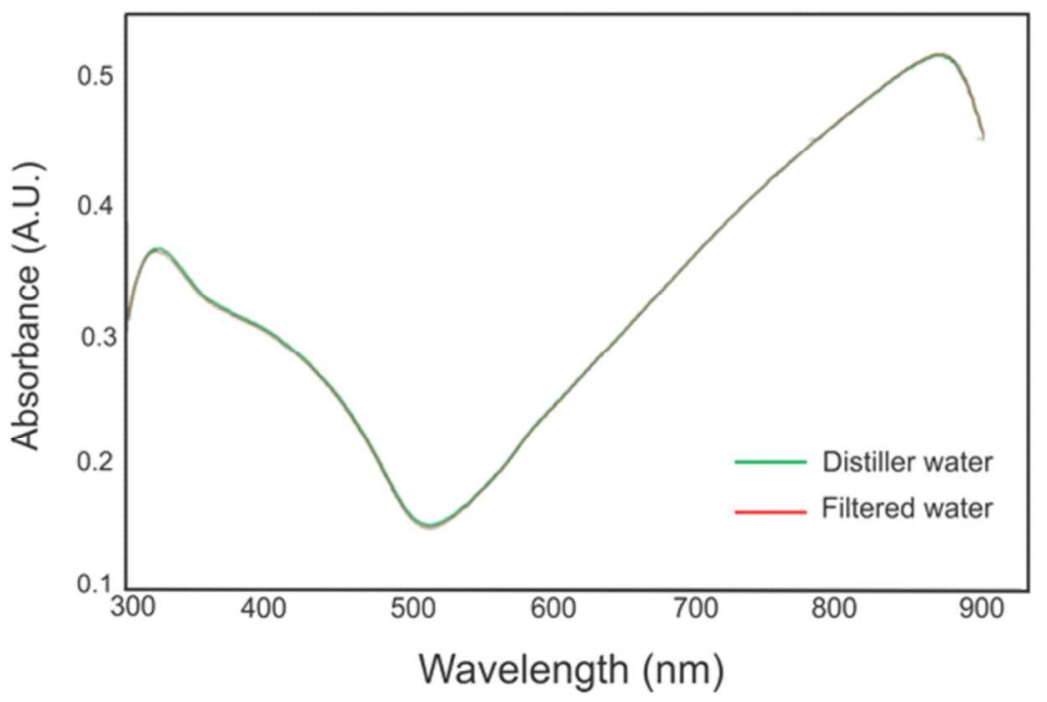

Figure S3. Absorbance spectrum for a gold surface before and after exposure to a water sample with mercury $(1000 \mathrm{ppb})$ that has been filtered by the pre-treatment system. No changes are observed in the absorbance. 